\title{
Photo-oxidation by laser pulse induced desorption of phthalocyanines
}

\author{
M. Holz ${ }^{\mathrm{a}}$, J. Wichmann ${ }^{\mathrm{a}}$, R. Mitrićb ${ }^{\mathrm{b}}$, L. Wöste ${ }^{\mathrm{a}}$, A. Lindinger ${ }^{\mathrm{a}, *}$ \\ ${ }^{a}$ Freie Universität Berlin, Institut für Experimentalphysik, Arnimallee 14, DE-14195 Berlin \\ ${ }^{b}$ Freie Universität Berlin, Institut für Theoretische Physik, Arnimallee 14, DE-14195 Berlin
}

\begin{abstract}
Photo-oxidation of iron(II)-phthalocyanine ( $\mathrm{PcFe}$ ) has been observed in matrix assisted laser desorption/ionization (MALDI) and laser desorption/ionization (LDI) and is interpreted by theoretical molecular dynamics simulations. The two ionization methods show different amounts of $\mu$-oxo-bridged PcFe-dimer and deliver evidence that MALDI produces less mechanical stress on the analyte. The typical proton-transfer in the MALDI-process does not occur which leads to the assumption of a released electron of the delocalized $\pi$-system.
\end{abstract}

(C) 2011 Published by Elsevier Ltd.

Keywords: MALDI, LDI, Phthalocyanine

\section{Introduction}

In the today's world phthalocyanines and their derivatives are widespread. Mainly used as dyes, they are also found in electronics, photo-dynamic therapy or modern optical devices. These manifold applications and their special physical and chemical properties have not only lead to numerous investigations on their optical, magnetic, and electric characteristics, but make them also very attractive in various research areas like crystallization and the usability as catalysts for $\mathrm{O}_{2}$ reduction in biological fuel cell applications or as molecular switches.[1, 2, 3, 4, 5] Most of the experimental studies have been performed in solution or in solid form. Nevertheless they are simply brought into the gas phase by laser pulse irradiation due to the high absorbance of light in the visible and near UV wavelength regime.[6] Therefore, Pcs are suitable matrix substances for matrix-assisted laser desorption/ionization (MALDI).

Phthalocyanines are $\pi$-conjugated macro-cyclic ligands and can coordinate metal elements like iron to so called metal phthalocyanines (PcMs). Some of them show the ability to adapt an oxygen atom if the present partial pressure of oxygen is high enough.[7] In the case of iron(II)-phthalocyanine ( $\mathrm{PcFe}$ ), several studies suggest that the additional oxygen atom binds to the central iron atom.[8]

15 This work reports about PcFe and its transformation into the gas phase via MALDI and laser desorption/ionization (LDI). Whereas in LDI the substance is directly excited, in MALDI the energy of the laser pulse is mostly absorbed by the matrix substance and transfers part of that energy to the analyte. The indirect

\footnotetext{
${ }^{*}$ Corresponding author

Email address: albrecht.lindinger@fu-berlin.de (A. Lindinger)
} 
energy transport has the advantage that the method can bring large fragile bio-molecules into the gas phase. In most cases, the matrix substances are organic acids and provide a proton to a basic residue of the analyte. In addition to the experimental results, molecular dynamics simulations have been utilized to support the interpretation of the presented data.

\section{Experimental}

All experiments have been performed with a commercial Bruker MALDI mass spectrometer (Reflex, Bruker Daltonics, Bremen, Germany). A short modification in the optical setup containing a dielectric mirror in the beam path has been made, which allows to irradiate the sample either with the built-in nitrogen laser (VSL-337ND, LSI) or ultra short laser pulses. The femtosecond laser setup (Kapteyn-Murmane Design; Odin, Quantronix, Darmstadt) provides $70 \mathrm{fs}$ laser pulses up to a pulse energy of $E=1 \mathrm{~mJ}$ at a central wavelength of $\lambda_{\mathrm{c}}=800 \mathrm{~nm}$. A detailed description can be found elsewhere. [9] The advantage of femtosecond laser pulses lies in the well defined beam profile and lower pulse-to-pulse deviation compared to the nitrogen laser.

For LDI-measurements, $50 \mathrm{mg} / \mathrm{ml}$ PcFe were solvated in a mixture of acetonitrile and water (3:2) and stirred for 30 minutes. $2 \mu \mathrm{l}$ of this mixture has been placed on each spot of the sample holder, dried with a heat gun for 15 minutes and cooled down below a fume hood for one hour. For MALDI-measurements $50 \mathrm{mg} / \mathrm{ml}$ of each $\alpha$-cyano-cinammic-acid and sinapinic acid has been dissolved in a mixture of acetonitrile and water (3:2) and $0.1 \mu \mathrm{l}$ trifluoracetic acid. The PcFe solution as described in the previous paragraph was again diluted in acetonitrile/water (3:2) in a ratio of 1:100, mixed with the matrix solution (1:1) and placed on the sample holder. The process of drying is the same as explained for LDI. The phthalocyanine substance was purchased from Alpha Aesar (Ward Hill MA, USA), all other chemicals have been ordered from Fluka (Buchs, Switzerland).

\section{Theoretical Methods}

The molecular structure has been calculated using an annealing procedure combined with molecular dynamics simulations at the semi-empirical AM1 level of theory.[10] The electronic structure was calculated with the MOPAC program package while the MD-simulation was performed with an in-house suite of programs.[11]

The simulations were started at a temperature $T=1000 \mathrm{~K}$ and the system has been exponentially cooled down to $T=0 \mathrm{~K}$ within $10 \mathrm{ps}$ of simulation time. The step size was chosen in the range of molecular vibrations to $1 \mathrm{fs}$. For a good statistics all structures have been carried out by varying the simulation parameters such as initial structure and temperature, as well as cooling time of the system.

The obtained results from this annealing procedure have been further optimized using DFT. For this purpose the ORCA-package has been used and the gradient corrected BP3LYP functional together with split valence plus polarization (SVP) basis sets for $\mathrm{H}$ and $\mathrm{C}$ and the triple-zeta valence plus polarization (TZVP) basis sets for $\mathrm{Fe}, \mathrm{N}$ and $\mathrm{O}$ have been applied. To estimate proton affinities, the basis set has been extended to double-zeta (DZ) for H and N. [12, 13, 14, 15]

\section{Results and Discussion}

\subsection{MALDI and LDI of [PcFe]}

All MALDI and LDI measurements were performed in positive ion mode. The MALDI mass spectrum in fig. 1 shows the typical pattern: Fragments of the analyte mixed with fragments of the matrix and cluster thereof at lower $\mathrm{m} / \mathrm{z}$-values and the analyte peak of $\mathrm{PcFe}$ at $\mathrm{m} / \mathrm{z}=568$. According to general MALDI mass spectra one would expect a protonated and maybe some cationized species. The exclusive occurrence of $[\mathrm{PcFe}]^{+}$suggests only a minor influence to charge transfer of the matrix substance. The observed side peaks in the mass spectrum reflect the isotopic abundances. In table 1 calculated proton affinities for different attacking sites on PcFe are listed. Following expression (2) in Ref. [16], the change of the molar enthalpy 


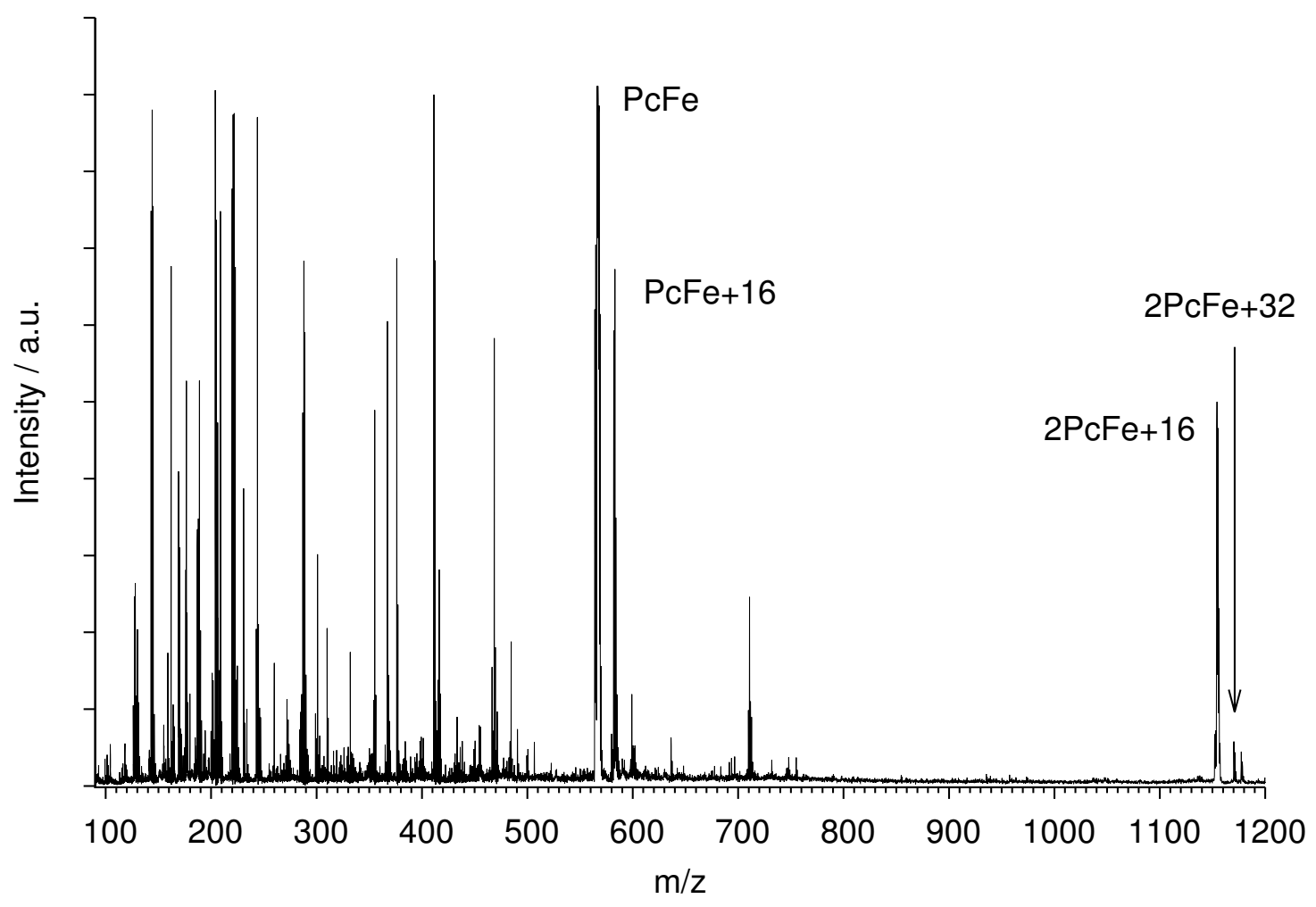

Fig. 1. MALDI mass spectrum of PcFe obtained with a pulse energy of $E=2 \mu \mathrm{J}$ : Clusters and fragments are caused by the matrix in the lower mass region. The analyte peak is found at $568 \mathrm{u}$ next to the oxidized species. At $1152 u$ the $\mu$-oxo bridged dimer can be found together with a doubly oxidized dimer at $1168 \mathrm{u}$. 


\begin{tabular}{cccc}
\hline Protonated atom & $\Delta E_{\mathrm{el}}^{0}$ & $\Delta E_{\mathrm{ZPE}}$ & $\Delta H_{298}$ \\
\hline N5 & -169.7 & 8.3 & -170.0 \\
C1 & -219.0 & 7.5 & -213.0 \\
C4 & -185.8 & 7.1 & -180.2 \\
\hline
\end{tabular}

Table 1. Energetic quantities for calculating the proton affinities of PcFe for different attacking sites. All values are given in $\mathrm{kcal} / \mathrm{mol}$. See fig. 3 for labeling.

for the reaction $[\mathrm{PcFe}]+\mathrm{H}^{+} \rightarrow[\mathrm{PcFe}+\mathrm{H}]^{+}$is given by $\Delta H_{298}=\Delta E_{\mathrm{el}}^{0}+\Delta E_{\mathrm{ZPE}}+\Delta E_{\mathrm{vib}}-\frac{5}{2} R T$. The first term, the difference in electronic energy $\Delta E_{\mathrm{el}}^{0}$ of reactants and product, is obtained by geometry optimizations. The change in energy associated with internal vibrations is the sum of the zero point energy $\Delta E_{\mathrm{ZPE}}$ and a correction term $\Delta E_{\mathrm{vib}}$. The latter accounts for a redistribution of the vibrational population as a function of temperature. Assuming that $\Delta E_{\mathrm{ZPE}} \gg \Delta E_{\mathrm{vib}}$, it has been neglected. The estimated proton affinities range from $\mathrm{PA}(\mathrm{PcFe})=170 \mathrm{kcal} / \mathrm{mol}$ to $213 \mathrm{kcal} / \mathrm{mol}$. These are relatively high values and a proton transfer reaction from the matrix to $[\mathrm{PcFe}]$ should be favorable. On the other hand, a delocalized electron of the $\pi$-system of the PcFe molecule can be easily detached by laser irradiation.

In the LDI mass spectrum (fig. 2) there are significantly less mass peaks in the low $\mathrm{m} / \mathrm{z}$ range. The lack of peaks (up to $\mathrm{m} / \mathrm{z}=450$ ) resulting from the matrix makes the identification of the parent and its corresponding fragments or clusters much easier. In this case one can identify three additional mass peaks, next to the parent ion, which are also visible in the MALDI mass spectrum. They are found at 584, 1152 and $1168 \mathrm{u}$. The first one has a shift of +16 mass units with respect to the analyte and suggests an attachment of either oxygen or a $-\mathrm{NH}_{2}$ group. The latter can be excluded, because no stable structures could be obtained from the performed geometry optimization calculations. The other two observed mass peaks can be interpreted as a PcFe-dimer with a $\mu$-oxo bridge between the two central iron atoms. The abundance of dimers is much higher in the MALDI spectrum which motivates the assumption of a soft release of the analyte into the gas phase. The matrix acts as a buffer and absorbs the kinetic energy of the molecules in the process of desorption. Moreover, other PcMs ( $\mathrm{PcCu}, \mathrm{PcZn}$ ) have been investigated for oxygen attachment, but it has been solely observed with PcFe.

\section{2. $[\mathrm{PcFe}]^{+}$and $[\mathrm{PcFe}]-\mathrm{O}^{+}$}

In fig. 3 all successfully geometry optimized configurations with minimal energy are shown and obtained structural parameters are summarized in table 2 . The electronic ground state of $[\mathrm{PcFe}]^{+}$is known as a ${ }^{4} A_{2 u}$ open shell system.[17] The [PcFe] ${ }^{+}$cation (a) exhibits a flat planar structure with $\mathrm{C}_{2 h}$ symmetry and is in agreement with recent theoretical results.[18] This optimized structure has been used as a basis for all further calculations.

The attachment of oxygen to $[\mathrm{PcFe}]^{+}$in (b) yields a distorted structure where the iron is displaced out of the plane, that is created by the nitrogen atoms of the pyrrole compounds, towards the oxygen, although the Pc configuration stays flat. In the accuracy of the calculation, the structure reveals a perfect $D_{4 v}$ symmetry.

\section{3. $[2 \mathrm{PcFe}]^{+}-\mathrm{O}$ and $[2 \mathrm{PcFe}]^{+}-\mathrm{O}_{2}$}

The dimer is formed in a reaction between a positively charged $\mathrm{PcFe}^{(I V)}-\mathrm{O}^{+}$and a neutral $\mathrm{PcFe}^{(I I I)}$, resulting in a $\left[\mathrm{PcFe}^{(I I I)}-\mathrm{O}-\mathrm{PcFe}^{(I I I)}\right]^{+}$-complex. Calculations of the $\mu$-oxo diiron, fig. 3 (c,d), lead to two different isomers. In structure (c) the iron atoms are coordinated in a square-pyramidal arrangement and the two phthalocyanines are rotated by $45^{\circ}$ relative to one another. The $\mathrm{Fe}-\mathrm{O}-\mathrm{Fe}$ ' bond shows a kink with an angle of $\theta_{F e-O-F e^{\prime}}=169.3^{\circ}$. The second conformer exhibits a parallel coordination of the PcFe-planes with an almost linear $\mathrm{Fe}-\mathrm{O}-\mathrm{Fe}$ ' bond system of $\theta_{\mathrm{Fe}-\mathrm{O}-\mathrm{Fe} e^{\prime}}=178.8^{\circ}$. The other structural parameters of (c,e) are very similar. UV-vis, NMR, IR and Mössbauer experiments support the existence of two stable isomers, but so far no gas phase data is available.[19, 8] Furthermore, X-ray crystallographic analysis of $\mu$-oxo diiron porphycene complexes data is present with Fe-O-Fe' angles of $178.7^{\circ}$ and $145.3^{\circ}$ and for $\mu$-oxo-bis[(1methylimidazole)-phthalocyaninatoirin(III)] of $175.1^{\circ}$.[20, 21] In both experiments rotated complexes are presented. 


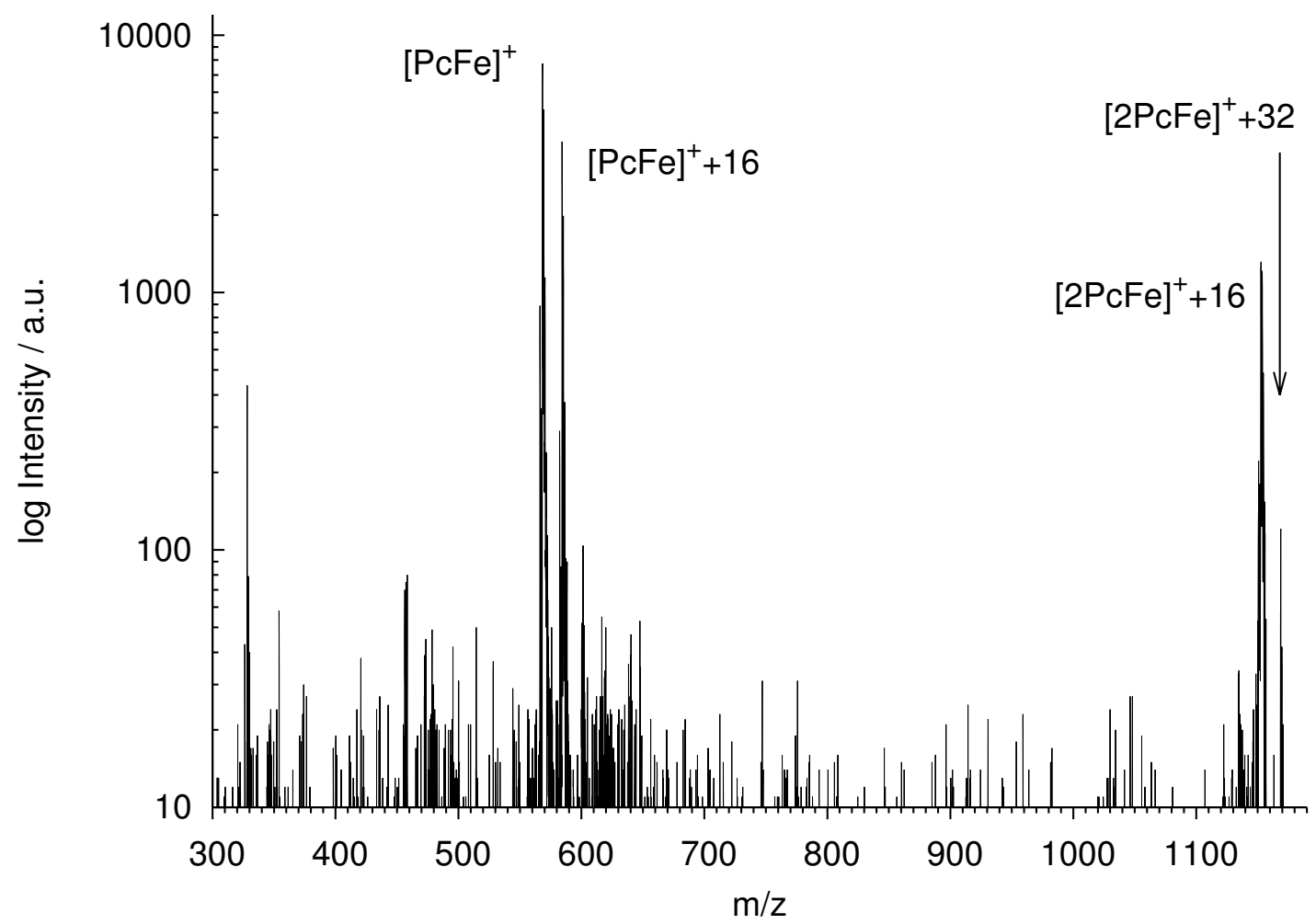

Fig. 2. LDI mass spectrum of PcFe obtained under the same conditions as the MALDI spectrum: At 568 u the ionized dye, the oxidized analyte at $584 \mathrm{u}$ and the $\mu$-oxo bridged dimer at $1152 \mathrm{u}$ is visible. A mass peak with a low intensity at $1168 \mathrm{u}$ which corresponds to two attached oxygen atoms can be observed.

\begin{tabular}{|c|c|c|c|c|c|c|}
\hline & {$[\mathrm{PcFe}]^{+}$} & {$[\mathrm{PcFe}-\mathrm{O}]^{+}$} & {$[2 \mathrm{PcFe}-\mathrm{O}]^{+}(\mathrm{c})$} & {$[2 \mathrm{PcFe}-\mathrm{O}]^{+}(\mathrm{e})$} & {$\left[2 \mathrm{PcFe}-\mathrm{O}_{2}\right]^{+}(\mathrm{d})$} & {$\left[2 \mathrm{PcFe}-\mathrm{O}_{2}\right]^{+}(\mathrm{f})$} \\
\hline$R_{\mathrm{N} 1-\mathrm{Fe}}$ & 1.944 & 1.962 & 1.983 & 1.983 & 1.988 & 1.981 \\
\hline$R_{\mathrm{N} 2-\mathrm{Fe}}$ & 1.952 & 1.962 & 1.983 & 1.984 & 1.988 & 1.980 \\
\hline$R_{\mathrm{N}^{\prime}-\mathrm{Fe}^{\prime}}$ & - & - & 1.982 & 1.983 & 1.970 & 1.979 \\
\hline$R_{\mathrm{N}^{\prime}-\mathrm{Fe}^{\prime}}$ & - & - & 1.984 & 1.983 & 1.970 & 1.959 \\
\hline$R_{\mathrm{Fe}-\mathrm{O}}$ & - & 1.614 & 1.799 & 1.799 & 1.779 & 1.846 \\
\hline$R_{\mathrm{O}-\mathrm{Fe}^{\prime}}$ & - & - & 1.798 & 1.801 & 1.955 & 1.747 \\
\hline$R_{\mathrm{Fe}^{\prime}-\mathrm{O}^{\prime}}$ & - & - & - & - & 1.658 & 1.813 \\
\hline$\theta_{\mathrm{N} 1-\mathrm{Fe}-\mathrm{N} 3}$ & 180 & 163.3 & 160.0 & 159.7 & 158.8 & 159.4 \\
\hline$\theta_{\mathrm{Fe}-\mathrm{O}-\mathrm{Fe}^{\prime}}$ & - & - & 169.3 & 178.8 & 179.2 & 179.5 \\
\hline$\theta_{\mathrm{N1}^{\prime}-\mathrm{Fe}^{\prime}-\mathrm{N} 3^{\prime}}$ & - & - & 159.7 & 159.8 & 176.5 & 171.2 \\
\hline
\end{tabular}

Table 2. Calculated parameters (bond length $R$ in $\AA$, bond angle $\theta$ in deg). Atom labels from fig.3, atoms of upper PcFe are denoted with'. 
An analogous picture is given for the doubly oxidized PcFe-dimer (d,f). Two isomeres have been obtained, but both with a nearly linear bonding along the Fe-O-Fe'-O' axis. Between structure (e) to (f) only minor changes in several bond lengths can be noticed. Dimer (d) instead shows significant modifications and result in a flat upper plane, similar to (a), induced by the additional ligand.

\section{Conclusion}

The catalyzed $[\mathrm{PcFe}]^{+}$has been observed for the first time in gas phase using MALDI and LDI. Theoretical calculations have shown, that the minimal energy $\mu$-oxo bridged dimer results in two different conformers. The oxygen has a strong influence on the central metal atom, but leads only to minor displacements in the atomic arrangement of the phthalocyanines. However, further calculations on a higher level of theory are necessary to estimate more accurate structural parameters. Although the gas-phase basicities are adequate, a protonation of $[\mathrm{PcFe}]$ in MALDI seems to be unfavored.

\section{References}

[1] C. M. Allen, W. M. Sharman, J. E. van Lier, Current status of phthalocyanines in the photodynamic therapy of cancer, J. Porphyrins Phthalocyanines 5 (2001) 161-169.

[2] R. Wagner, J. Lindsey, J. Seth, V. Palaniappan, D. Bocian, Molecular optoelectronic gates, J. Am. Chem. Soc 118 (1996) 39963997.

[3] H. Moons, Ł. Ł. apok, A. Loas, S. van Doorslaer, S. M. Gorun, Synthesis, x-ray structure, magnetic resonance, and dft analysis of a soluble copper(ii) phthalocyanine lacking c-h bonds, Inorg. Chem. 49 (2010) 8779-8789.

[4] E. H. Yu, S. Cheng, B. E. Logan, K. Scott, Electrochemical reduction of oxygen with iron phthalocyanine in neutral media, J. Appl. Electrochem. 39 (2009) 705-711.

[5] P. Liljeroth, J. Repp, G. Meyer, Current-induced hydrogen tautomerization and conductance switching of naphthalocyanine molecules, Science 317 (2007) 1203-1206.

[6] D. Dini, H. Hanack, Phthalocyanines as materials for advanced technologies: some examples, J. Porphyrins Phthalocyanines 8 (2004) 915-933.

[7] V. I. Nemykin, V. Y. Cherni, S. V. Volkov, N. I. Bundina, O. L. Kaliya, V. D. Li, E. A. Luckyantes, Further studies on the oxidation state of iron in $\mu$-oxo dimeric phthalocyanine complexes, J. Porphyrins Phthalocyanines 3 (1999) 87-98.

[8] A. A. Tanaka, C.Fierro, D.Scherson, E.B.Yeager, Electrocatalytic Aspects of Iron Phthalocyanine and Its $\mu$-Oxo Derivatives Dispersed on High Surface Area Carbon, J. Phys. Chem. 91 (1987) 3799-3807.

[9] J. Wichmann, C. Lupulescu, L. Wöste, A. Lindinger, Matrix-assisted laser desorption/ionization by using femtosecond laser pulses in the near-infrared wavelength regime, Rapid Commun. Mass Spectrom. 23 (2009) 1105-1108.

[10] M. Dewar, E. Zoebisch, E. Healy, J. Stewart, AM1: A new general purpose quantum mechanical model, J. Am. Chem. Soc. 107 (1985) 3902-3909.

[11] J. J. P. Stewart, MOPAC, stewart Computational Chemistry, Fujitsu Ltd. 2001.

[12] F. Neese, The orca program system, Wiley Interdisciplinary Reviews: Computational Molecular Science 2 (1) (2012) 73-78, (Version 3.0).

[13] A. D. Becke, Density-functional thermochemistry. iii. the role of exact exchange, J. Chem. Phys. 98 (1993) 5648-5652.

[14] P. J. Stephens, F. J. Devlin, C. F. Chabalowski, M. J. Frisch, Ab initio calculation of vibrational absorption and circular dichroism spectra using density functional force fields, J. Phys Chem. 98 (1994) 11623-11627.

[15] A. Schäfer, H. Horn, R. Ahlrichs, Fully optimized contracted Gaussian-basis sets for atoms Li to Kr, J. Chem. Phys. 97 (1992) 2571-2577.

[16] D. A. Dixon, J. L. Gole, A. Komornicki, Absolute proton affinities of lithium dimer, sodium dimer, lithium hydride, and sodium hydride, J. Phys. Chem. 92 (8) (1988) 2134-2136.

[17] M.-S. Liao, S. Scheiner, Electronic structure and bonding in metal phthalocyanines, metal=fe, co, ni, cu, zn, mg, J. Chem. Phys. 114 (22) (2001) 9780-9791.

[18] M. Sumimoto, Y. Kawashima, K. Hori, H. Fujimoto, Theoretical investigation of the molecular and electronic structures and excitation spectra of iron phthalocyanine and its derivatives, fepc and fepcl ${ }_{n}\left(1=\right.$ py, $\left.\mathrm{cn}^{-} ; n=1,2\right)$, Dalton Trans. (2009) 57375746

[19] N. Mekhryakova, T. Gulina, V. Yu, N. Bundina, O. Kaliya, E. Luk'yanets, Reaction of Iron Phthalocyanine with Oxygen: Critical Survey and New Data on the Structure of the Forming PcFe(II) $\mu$-Oxo Dimer, Russ. J. Gen. Chem. 71 (2001) 570-590.

[20] M. Lausmann, I. Zimmer, J. Lex, H. Lueken, K. Wieghardt, E. Vogel, $\mu$-oxodiiron(iii) complexes of porphycenes", Angew. Chem. Int. Ed. Engl. 33 (7) (1994) 736-739.

[21] C. Ercolani, F. Monacelli, S. Dzugan, V. L. Goedken, G. Pennesi, G. Rossi, X-ray crystal structure of [small micro]-oxo-bis[(1methylimidazole)-phthalocyaninatoiron(iii)] and comments on the molecular structure and chemistry of oxo-bridged iron phthalocyaninate dimers, J. Chem. Soc., Dalton Trans. (1991) 1309-1315. 
(a)

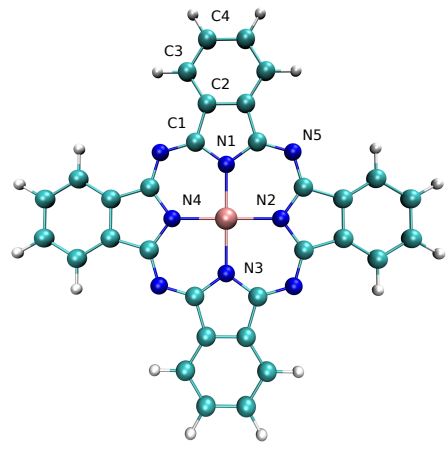

(c)
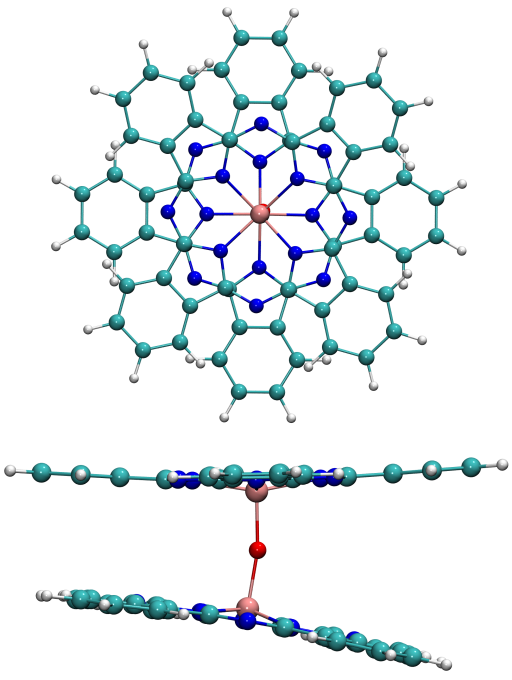

(e)

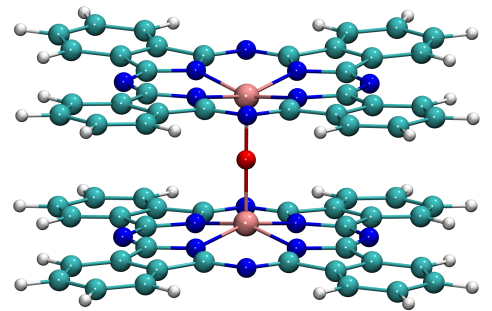

(b)

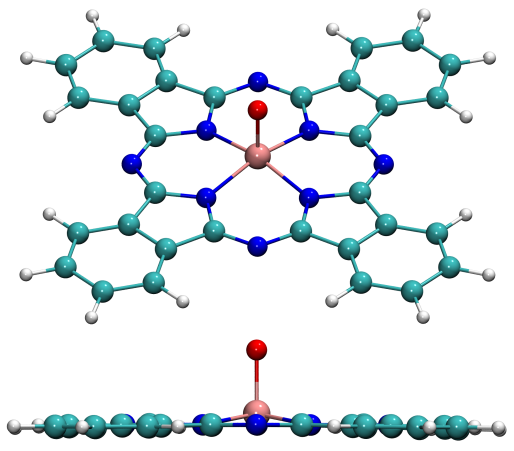

(d)
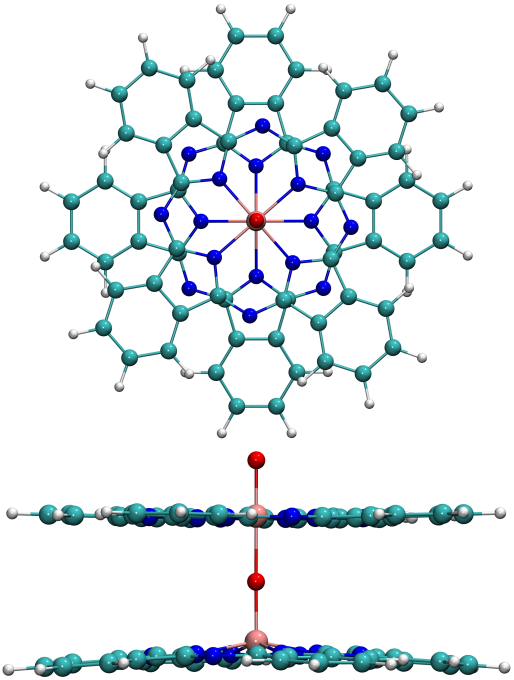

(f)

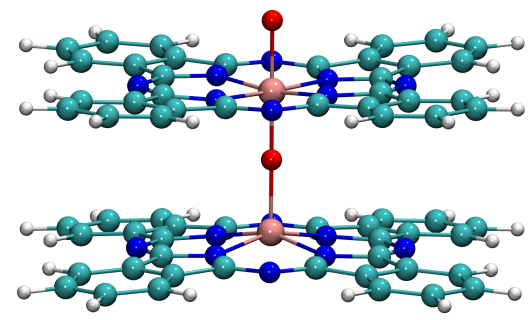

Fig. 3. Geometry optimized structures of minimal energy in tilted top and side view of (a) Iron(II)-Phthalocyanine, (b) PcFe-O, (c,e) dimer structure $\mathrm{PcFe}-\mathrm{O}-\mathrm{PcFe}$, (d,f) doubly oxidized dimer structure $\mathrm{PcFe}-\mathrm{O}-\mathrm{PcFe}-\mathrm{O}$. For details see text. 